\title{
Histopathological changes caused by Enteromyxum leei infection in farmed sea bream Sparus aurata
}

\author{
R. Fleurance ${ }^{1}$, C. Sauvegrain ${ }^{2}$, A. Marques ${ }^{3}$, A. Le Breton ${ }^{4}$, C. Guereaud ${ }^{1}$, \\ Y. Cherel ${ }^{1}$, M. Wyers ${ }^{1, *}$ \\ ${ }^{1}$ Department of Veterinary Pathology, UMR 703 INRA/ENVN, Nantes Veterinary School, BP 40706, 44307 Nantes cedex 03 , \\ France \\ ${ }^{2}$ Aquanord, Terre des marins, 59820 Gravelines, France \\ ${ }^{3}$ DRIM Dept BEE, UM2, case 080 Université Montpellier, 34095 Montpellier cedex 5, France \\ ${ }^{4}$ Fish Health Consultant, 31330 Grenade sur Garonne, France
}

\begin{abstract}
Histological examinations were carried out on the stomach, pyloric caeca and 4 different parts of the intestine, as well as the rectum, hepatopancreas, gall bladder and spleen of 52 sea bream Sparus aurata spontaneously infected by Enteromyxum leei. Fifteen fish from a non-infected farm were included as a control. Clinical signs appeared only in extensively and severely infected fish. We observed Enteromyxum leei almost exclusively in the intestinal tract, and very rarely in the intrahepatic biliary ducts or gall bladder. We observed heavily infected intestinal villi adjacent to parasite-free villi. Histological changes indicated a parasite infection gradually extending from villus to villus, originating from an initial limited infected area probably located in the rectum. The parasite forms were exclusively pansporoblasts located along the epithelial basement membrane. Periodic acid-Schiff (PAS)-Alcian blue was the most useful histological stain for identifying the parasite and characterising the degree of intestinal infection. We observed severe enteritis in infected fish, with inflammatory cell infiltration and sclerosis of the lamina propria. The number of goblet cells was considerably and significantly decreased in heavily infected fish. The intestines of 4 of the 5 survivor fish were totally free of parasites and showed severe chronic enteritis with a regenerative epithelium, suggesting that an acquired immune process may spontaneously eliminate parasites.
\end{abstract}

KEY WORDS: Enteromyxum leei $\cdot$ Myxidiosis $\cdot$ Parasitic enteritis $\cdot$ Histopathology $\cdot$ Sparus aurata

Resale or republication not permitted without written consent of the publisher

\section{INTRODUCTION}

Myxidiosis is caused by a myxozoan parasite, Enteromyxum leei (formerly Myxidium leei), and is one of the most severe parasitic diseases which is currently expanding in cultured Mediterranean fish species (Le Breton \& Marques 1995). The parasite was first described by Diamant (1992) in cultured sea bream Sparus aurata from southern Cyprus. Since 1991-1992, serious losses to cultured Mediterranean sparids have been attributed to myxidiosis (Diamant 1992, Le Breton \& Marques 1995, Sakiti et al. 1996). More recently, Padros et al. (2001) identified Enteromyxum leei infection in 25 different fish species reared in an exhibition aquarium. Recently, the sea bass Dicentrachus labrax has been recognised as being sensitive to this parasite in cultured conditions.

Myxozoans were first classified as protists, but their classification has become a matter of some controversy since the description of their multi-cellular spores (Grassé \& Lavette 1978). Currently, myxozoan parasites are attributed to the phylum Metazoa. To our knowledge, the genus Enteromyxum includes 3 species of histozoic and enteritic myxozoan parasites in marine cultured fish: E. leei from sea breams, with a wide host specificity, E. scophthalmi from cultured turbots (Palenzuela et al. 2002) and E. fugu (formerly Myxidium fugu) from cultured tiger puffer (Yanagida et al. 2004). 
Many myxozoan species require an actinosporean form as an alternate life cycle stage. The current thinking on the myxozoan life cycle suggests an indirect cycle with an alternate myxosporean form in fish and an actinosporean form in oligochetes (Wolf \& Markiw 1984, Benajiba \& Marques 1993, El-Matbouli \& Hoffmann 1993, Lom \& Dykova 1995, Tarer et al. 1996). While the alternate host and the actinosporean form of Enteromyxum leei have not yet been identified, Diamant (1997) has demonstrated experimentally a direct fish to fish transmission in sea bream without the participation of an alternate host.

In the case studied here, a fish farm in Northern France (Gravelines) suffered a high mortality in its cultured sea bream population. The affected fish became emaciated, their skin colour darkened, and they had sunken eyes and cutaneous lesions and were found swimming near the surface. The dying sea breams had a pale and soft liver and enlarged gall bladder. Scrapings from the rectal mucosa of the fish from affected pools revealed the presence of Enteromyxum leei, which was then confirmed by histological examination of the rectum.

This study describes the histopathological changes caused by Enteromyxum leei in the digestive tract of spontaneously infected sea bream. We focus on lesions in the digestive and digestive-associated systems (hepatopancreas) as well as in the spleen, and histologically describe the morphological characteristics of the parasites.

\section{MATERIALS AND METHODS}

Animals. In the early summer of 2002, we obtained tissue samples from 67 farmed sea bream Sparus auratus of the same age. The fish were divided into 5 sample groups. Four of the groups were derived from recognised Enteromyxum leei-affected pools at a fish farm in northern France. The fish from this farm were classified into 4 groups according to the following characteristics:

- AH ('Apparently healthy') group: 15 fish (weight: $702.2 \pm 115.1 \mathrm{~g}$; length: $30.7 \pm 1.3 \mathrm{~cm}$ ) from an affected pool, but showing no cutaneous lesions, emaciation or any other clinical signs

- SS ('Slightly sick') group: 16 fish (weight: $437.2 \pm$ $94.2 \mathrm{~g}$; length: $29.5 \pm 1.7 \mathrm{~cm}$ ) with clinical signs of darkening, swollen abdomen, fin necrosis and anus tumefaction, but no emaciation

- MO ('Moribund') group: 16 fish (weight: $488.0 \pm$ $99.3 \mathrm{~g}$; length: $29.3 \pm 2.0 \mathrm{~cm}$ ) at the terminal stage of the disease, with emaciation, darkening, swollen abdomen, fin necrosis, anus tumefaction, haemorrhage and sunken eyes
- SV ('Survivor') group: 5 surviving fish from a pool that had 2 previous Enteromyxum leei outbreaks causing death, occurring $1 \mathrm{yr}$ and 6 mo before the sampling.

The fifth group was a control (C) group comprising 15 fish from a fish farm recognised as being free of any Enteromyxum leei infection.

Histological samples. All the fish were killed using a standard acceptable method (Close et al. 1997). The entire digestive tract, from the stomach to the rectum, including the hepatopancreas with the gall bladder and spleen, was immediately fixed for histological study. The stomach, pyloric caeca, rectum and gall bladder were longitudinally sectioned. The entire length of the rectum was examined.

In most of the fish (10 from the AH, 14 from the SS, 14 from the MO and all 15 from the C group), several transversal sections were microscopically examined (i.e. 'transversally cut intestine' protocol) in 4 different areas of the intestine corresponding to 1/8 ('proximal'), 1/4 ('mid-proximal'), 3/4 ('mid-distal') and 7/8 ('distal') of the distance along the entire intestinal tract.

In 14 other fish from the $\mathrm{AH}(\mathrm{n}=5), \mathrm{SS}(\mathrm{n}=2), \mathrm{MO}$ ( $\mathrm{n}=2$ ) and SV (n = 5) groups, a 'longitudinally cut intestine' protocol was applied: the entire intestine was examined by means of longitudinal sections to determine the severity of infection along the intestinal tract and focus on the transitions between infected and noninfected areas.

Histological analysis. All the tissues were fixed in $10 \%$ neutral buffered formalin and processed using standard procedures for embedded and sectioned paraffin samples. Sections of approximately $4 \mu \mathrm{m}$ thickness were stained using HES (haematoxylin, eosin and saffron) and special stains, including periodic acid-Schiff (PAS)-Alcian blue and Gram methods. Spleen samples were stained with Perls staining to reveal ferric pigments.

An intestinal section was considered positive (i.e. infected) only if mature spores and/or pansporoblasts could be observed inside the digestive mucosa. The presence of spores in the lumen alone did not indicate that the section was positive. A digestive tissue section was considered positive if at least one of the histological sections of the corresponding area was infected.

Quantitative morphometric analysis. All histopathological studies were performed using standard procedures with uniform conditions of fixation and staining of $4 \mu \mathrm{m}$ sections with PAS-Alcian blue.

Quantitative morphometric analysis of the number of parasites per unit length of gut mucosa did not yield acceptable repeatability in individual fish, within groups or between groups.

The number of goblet cells (mucus cells) per $100 \mu \mathrm{m}$ of infected intestinal mucosa in the intestine of 24 
affected fish (PAS-Alcian blue stained histoslides; Nikon Analysing system LUCIA G) was quantified morphometrically and compared with the non-infected intestinal mucosa of fish from the C group ( $\mathrm{n}=15)$. For each group, 500 to 1000 mucus cells were counted at the level of the enterocyte cilia, including all the mucus cells observed along the corresponding length of intestinal epithelium.

Statistical analysis. The parasitic distribution in different parts of intestine among groups $(\mathrm{AH}, \mathrm{SS}$ and $\mathrm{MO}$ ) was statistically compared using the chi-squared test.

The number of goblet cells per $100 \mu \mathrm{m}$ of intestinal mucosa in infected fish was statistically compared to that in control fish. Normality and homogeneity of variances were tested using Kolmogorov-Lilliefors and Bartlett tests. Depending on the results, a Student $t$ test or a Mann-Whitney test was performed to evaluate the statistically significant differences between infected and non-infected fish.

All analyses were performed using SAS Enterprise Guide V2 (2.0.0.417, SAS Institute). A p value of 0.05 was used to define significant differences between groups.

\section{RESULTS}

\section{Enteromyxum leei at the histological level}

Enteromyxum leei were observed almost exclusively in the intestinal tract, being present from the pyloric caeca to the rectum area. We occasionally found some parasites in the gall bladder epithelium, and also in the intra-hepatic biliary ducts of a few of the most severely affected fish. Even in the most heavily infected fish of the different groups, we never observed parasite infection of the gastric mucosa, although spores could be seen in the gastric lumen. All fish in the control group were free of parasites.

In the mucosa, parasites were observed exclusively in the epithelium and seemed to be located between the epithelial cells in an intercellular space generally located close to the basement membrane. The lamina propria was always free of parasites (Figs. $1 \& 2$ ).

In the present study, we observed the pansporoblast morphological stage, which consists of a large, round and vacuolated element that is optically clear and which varies up to $40 \mu \mathrm{m}$ in diameter. It seems to be located outside the cells, usually between the basal parts of the enterocytes (Fig. 2). Although pansporoblasts can be identified by HES staining, the sporoblastic structure is difficult to analyse (Fig. 2). After PASAlcian blue staining, the pansporoblasts appear as pseudo-cysts bounded by a thin magenta-stained membrane and containing varying amounts of sporoblasts and spores uniformly stained magenta (Fig. 3). By contrast, the mucus cells exhibit a blue-stained apical cytoplasm that can be clearly differentiated from the parasitic elements (Fig. 3). Immature pansporoblasts contain varying amounts of cellular constituents. Mature pansporoblasts each contain 2 spores and 2 cells having an unknown role (occurring as 'cells in a cell') (Fig. 3). The spores are stained magenta with PASAlcian blue and are of consistent shape and size $(14.10 \pm 1.42 \mu \mathrm{m}$ long and $7.75 \pm 0.66 \mu \mathrm{m}$ wide, $\mathrm{n}=50)$. Each spore contains 2 polar capsules $(7.06 \pm 0.54 \mu \mathrm{m}$ long, $\mathrm{n}=100$ ) located at the opposite extremities of the spore, which are stained dark blue by the Gram staining technique (Fig. 4). They contain a polar filament that is picked out weakly with Gram staining (Fig. 4).

\section{Comparative study of the parasitic infection in the affected groups}

Table 1 shows the locations of the parasites found in $\mathrm{AH}, \mathrm{SS}$ and $\mathrm{MO}$ fish sampled by 4 transversal intestinal sections.

In the $\mathrm{AH}$ group of fish from a recognised affected pool, 4 of the 10 fish appeared to be free of any parasite infection. In the 6 remaining infected sea bream, the pyloric caeca were always free of parasites, with the proximal parts of the intestine being mildly affected ( 2 out of 6 ), while the mid-proximal (4 out of 6 ), mid-distal (4 out of 6 ) and distal parts (4 out of 6 ) and the rectum (5 out of 6 ) were moderately affected. None of the fish exhibited infection in all parts of their intestine. Four of the 6 infected displayed a massive and diffuse parasite infection of the rectum. This rectal infection was always associated with infection in one or more posterior parts of the intestine. One fish was mainly infected in the anterior parts of the intestine, with no parasites being found in the rectal mucosa. The other infected fish had a more extensive infection in the anterior parts of the intestine (proximal, mid-proximal and mid-distal parts) and a localised infection in the rectum. The longitudinally embedded gut samples allowed us to measure the respective lengths of safe and infected parts in the mucosa of 3 fish. One fish had a parasite infection present in $23 \%$ of the intestine length, with 3 separate parasitic foci in the distal gut and rectum. A second fish had only 1 distal intestinal parasitic focus corresponding to about $4 \%$ of the intestine length, and no parasitic focus in the rectum. In the third fish, $48 \%$ of the intestine length was infected, with 3 separate parasitic foci in the distal part of the gut and in the rectum. In all 3 fish, the pyloric caeca and proximal parts of the intestine were unaffected. Pyloric caeca and proximal intestine in the AH group 

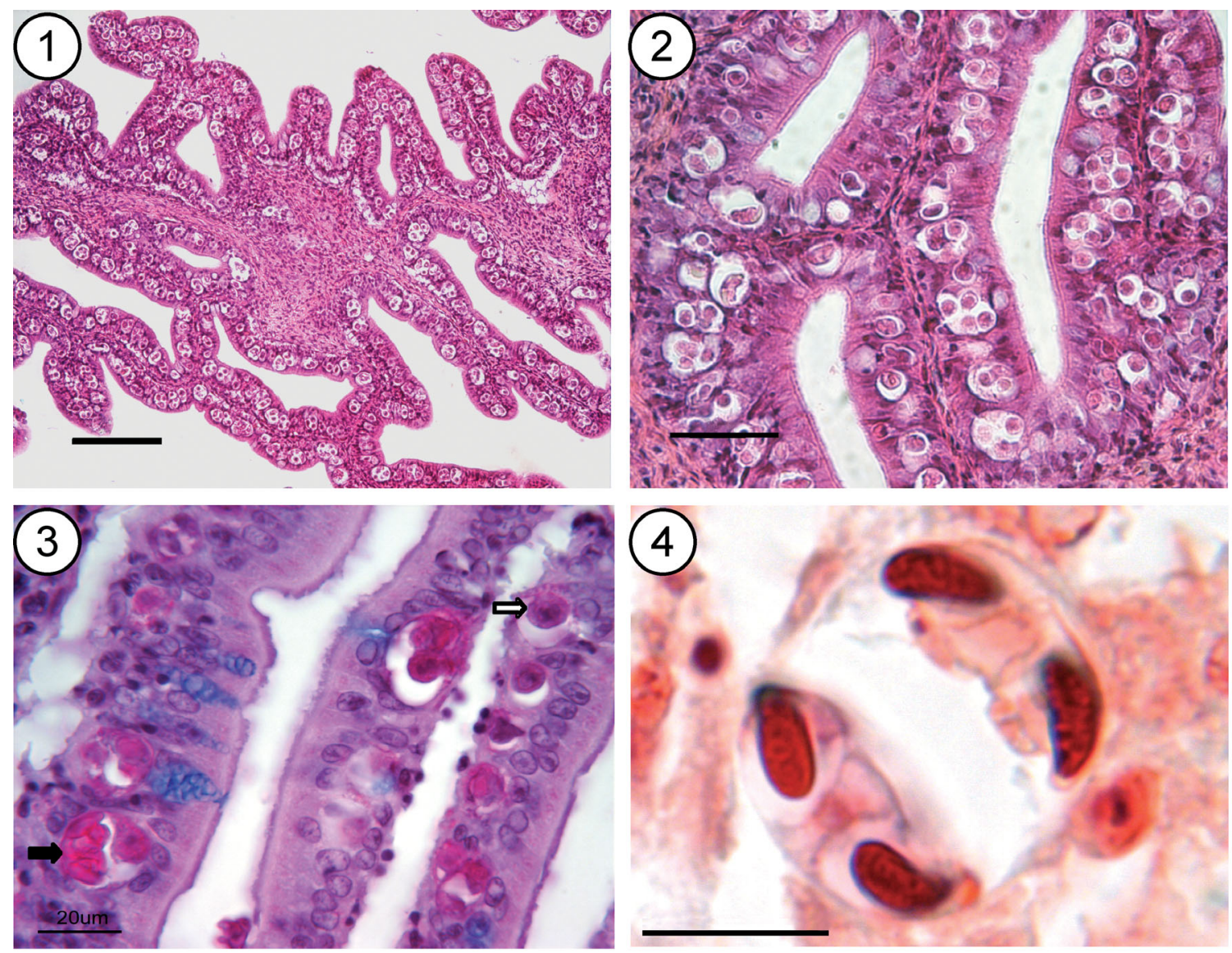

Figs. 1 to 4. Enteromyxum leei infecting Sparus aurata. Fig. 1. Intestine of an infected sea bream: Pansporoblasts are located only in the intestinal epithelium. Infestation is severe, with only a few mononucleated inflammatory cells in the lamina propria. There is a focal detachment of the epithelium from the underlying lamina propria. Haematoxylin, eosin and saffron (HES) staining. Scale bar $=100 \mu \mathrm{m}$. Fig. 2. Intestine of a sea bream heavily infected by E. leei. Numerous pansporoblasts located close to the basement membrane make up an optically empty vacuolated constituent, containing few parasitic cells. The palisade-like arrangement of enterocytes is lost in cases of severe infestation, leading to an epithelium with pseudo-stratified appearance. The nuclei of enterocytes are almost always displaced to the apical pole of the cells. HES. Scale bar $=40 \mu \mathrm{m}$. Fig. 3 . Intestine of an infected sea bream. A pansporoblast is an optically empty vacuolated constituent, stained magenta by periodic acid-Schiff (PAS)-Alcian blue. Different stages of spore formation can be observed here: the white arrow shows a single sporoblast in a pansporoblast. To the left of this arrow a pansporoblast containing 2 sporoblasts can be seen, one of which has started to divide into several cells by internal cleavage resulting in 'cell in a cell' formation. The black arrow shows spore formation with a mature spore and a sporoblast. Spores are stained magenta with PAS-Alcian blue; goblet cells are stained blue. There is a detachment of the epithelium from the underlying lamina propria. Scale bar $=20 \mu \mathrm{m}$. Fig. 4. Spores of E. leei in a pansporoblast in the intestine of a sea bream. Each spore is composed of 2 polar capsules, stained dark blue by the Gram method, and a vacuolated cell weakly stained in the centre of the spore corresponding to the sporoplasm. Each polar capsule contains a polar filament. Gram stain. Scale bar $=10 \mu \mathrm{m}$

are significantly less frequently infected than the other distal parts $(\mathrm{p} \leq 0.01)$.

In the SS group of fish $(\mathrm{n}=16)$ exhibiting clinical signs of the disease but without emaciation, all fish were affected by Enteromyxum leei (Table 1). Among the 14 fish included in the 'transversally cut intestine' protocol, only 8 of the 84 examined areas were free of parasites. One fish had parasites only in the proximal parts of the intestine, while the remaining 13 fish were heavily affected from pyloric caeca to rectum. All parts were similarly infected: pyloric caeca (11 out of 13), proximal intestine (13 out of 13), mid-proximal intestine (11 out of 13), mid-distal intestine (12 out of 13), distal intestine (13 out of 13) and rectum (13 out of 13). Pyloric caeca and proximal intestine in the SS group were significantly more frequently infected compared to the AH group ( $\mathrm{p} \leq 0.05$ and $\mathrm{p} \leq 0.01$, respectively). Fish from the SS group show a significantly more extensive parasitic distribution compared to group $\mathrm{AH}$, with 76 infected areas out of the total of 84 observed areas $(p \leq 0.0001)$. A diffuse parasitic infection of the intestinal tract was confirmed in the 2 
Table 1. Location of parasites in fish included in 'transversally cut intestine protocol' (for details see 'Materials and methods'). AH: apparently healthy; SS: slightly sick; MO: moribund fishes; 0: digestive area free of parasites (grey shaded); +: digestive area positive for parasites; ( ): number of affected sections in the total number of examined sections

\begin{tabular}{|c|c|c|c|c|c|c|}
\hline \multirow[t]{2}{*}{ Fish } & \multirow{2}{*}{$\begin{array}{l}\text { Pyloric } \\
\text { caeca }\end{array}$} & \multicolumn{4}{|c|}{ Intestine } & \multirow{2}{*}{ Rectum } \\
\hline & & Proximal & Mid-proximal & Mid-distal & Distal & \\
\hline \multicolumn{7}{|c|}{ AH group } \\
\hline 1 & 0 & $0(0 / 3)$ & $0(0 / 3)$ & $0(0 / 3)$ & $0(0 / 3)$ & 0 \\
\hline 2 & 0 & $0(0 / 3)$ & $+(3 / 3)$ & $+(3 / 3)$ & $+(3 / 3)$ & + \\
\hline 3 & 0 & $+(3 / 3)$ & $+(3 / 3)$ & $0(0 / 3)$ & $0(0 / 3)$ & 0 \\
\hline 4 & 0 & $0(0 / 3)$ & $0(0 / 3)$ & $0(0 / 3)$ & $0(0 / 3)$ & 0 \\
\hline 5 & 0 & $0(0 / 3)$ & $0(0 / 3)$ & $+(3 / 3)$ & $+(3 / 3)$ & + \\
\hline 6 & 0 & $0(0 / 3)$ & $0(0 / 3)$ & $0(0 / 3)$ & $0(0 / 3)$ & 0 \\
\hline 7 & 0 & $0(0 / 3)$ & $0(0 / 3)$ & $0(0 / 3)$ & $+(3 / 3)$ & + \\
\hline 8 & 0 & $+(3 / 3)$ & $+(3 / 3)$ & $+(2 / 3)$ & $0(0 / 3)$ & + \\
\hline 9 & 0 & $0(0 / 3)$ & $0(0 / 3)$ & $0(0 / 3)$ & $0(0 / 3)$ & 0 \\
\hline 10 & 0 & $0(0 / 3)$ & $+(3 / 3)$ & $+(3 / 3)$ & $+(3 / 3)$ & + \\
\hline \multicolumn{7}{|c|}{ SS qroup } \\
\hline 1 & + & $+(2 / 2)$ & $+(2 / 2)$ & $+(3 / 3)$ & $+(3 / 3)$ & + \\
\hline 2 & + & $+(2 / 2)$ & $+(3 / 3)$ & $+(3 / 3)$ & $+(3 / 3)$ & + \\
\hline 3 & + & $+(1 / 1)$ & $+(2 / 2)$ & $+(2 / 3)$ & $+(2 / 2)$ & + \\
\hline 4 & + & $+(1 / 3)$ & $+(3 / 3)$ & $+(2 / 2)$ & $+(1 / 2)$ & + \\
\hline 5 & + & $+(2 / 2)$ & $+(3 / 3)$ & $+(1 / 3)$ & $+(3 / 3)$ & + \\
\hline 6 & + & $+(1 / 2)$ & $0(0 / 3)$ & $0(0 / 3)$ & $+(2 / 2)$ & + \\
\hline 7 & 0 & $+(2 / 2)$ & $+(3 / 3)$ & $+(3 / 3)$ & $+(3 / 3)$ & + \\
\hline 8 & + & $+(3 / 3)$ & $0(0 / 3)$ & $+(3 / 3)$ & $+(3 / 3)$ & + \\
\hline 9 & 0 & $+(1 / 2)$ & $+(3 / 3)$ & $0(0 / 3)$ & $0(0 / 3)$ & 0 \\
\hline 10 & + & $+(2 / 2)$ & $+(2 / 2)$ & $+(3 / 3)$ & $+(3 / 3)$ & + \\
\hline 11 & 0 & $+(2 / 2)$ & $+(3 / 3)$ & $+(3 / 3)$ & $+(1 / 2)$ & + \\
\hline 12 & + & $+(2 / 2)$ & $+(2 / 2)$ & $+(3 / 3)$ & $+(2 / 2)$ & + \\
\hline 13 & + & $+(2 / 2)$ & $+(3 / 3)$ & $+(3 / 3)$ & $+(3 / 3)$ & + \\
\hline 14 & + & $+(2 / 2)$ & $+(2 / 2)$ & $+(2 / 2)$ & $+(2 / 2)$ & + \\
\hline \multicolumn{7}{|c|}{ MO group } \\
\hline 1 & 0 & $0(0 / 2)$ & $0(0 / 2)$ & $+(2 / 2)$ & $0(0 / 2)$ & + \\
\hline 2 & + & $+(2 / 2)$ & $+(2 / 2)$ & $+(2 / 2)$ & $+(2 / 2)$ & + \\
\hline 3 & + & $+(2 / 2)$ & $+(2 / 2)$ & $+(2 / 2)$ & $+(1 / 2)$ & + \\
\hline 4 & + & $+(2 / 2)$ & $+(2 / 2)$ & $+(2 / 2)$ & $+(2 / 2)$ & + \\
\hline 5 & + & $+(2 / 2)$ & $+(3 / 3)$ & $+(3 / 3)$ & $+(2 / 2)$ & + \\
\hline 6 & + & $0(0 / 1)$ & $0(0 / 2)$ & $+(1 / 2)$ & $+(2 / 2)$ & + \\
\hline 7 & + & $+(2 / 2)$ & $+(1 / 2)$ & $+(2 / 2)$ & $+(1 / 2)$ & + \\
\hline 8 & + & $+(2 / 2)$ & $+(2 / 2)$ & $+(2 / 2)$ & $+(1 / 1)$ & + \\
\hline 9 & + & $+(2 / 2)$ & $+(2 / 2)$ & $+(2 / 2)$ & $+(2 / 2)$ & + \\
\hline 10 & + & $+(1 / 1)$ & $+(1 / 1)$ & $+(2 / 2)$ & $+(1 / 1)$ & + \\
\hline 11 & + & $+(2 / 2)$ & $+(2 / 2)$ & $+(2 / 2)$ & $+(2 / 2)$ & + \\
\hline 12 & + & $+(2 / 2)$ & $+(2 / 2)$ & $+(2 / 2)$ & $+(2 / 2)$ & + \\
\hline 13 & 0 & $+(1 / 2)$ & $+(2 / 2)$ & $+(2 / 2)$ & $0(0 / 2)$ & 0 \\
\hline 14 & + & $+(1 / 1)$ & $+(2 / 2)$ & $+(2 / 2)$ & $+(2 / 2)$ & + \\
\hline
\end{tabular}

caeca. In the 3 remaining fish, 1 had only one infected area in the mid-distal intestine. In the second fish, the pyloric caeca, the distal parts of the intestine and the rectum were all infected, and the third fish was infected in the proximal and midproximal intestine. In the MO group, the pyloric caeca, proximal and middistal intestine are significantly more frequently infected than in the $\mathrm{AH}$ group ( $\mathrm{p} \leq 0.01, \mathrm{p} \leq 0.05$ and $\mathrm{p} \leq 0.05$, respectively). Fish from the MO group show a significantly more extensive parasitic distribution, with 75 infected areas out of the total of 84 observed areas ( $\mathrm{p} \leq 0.0001)$ compared with group $\mathrm{AH}$. There is no statistical difference in the total number of infected areas between SS and MO groups. The 2 fish included in the 'longitudinally cut intestine' protocol showed a diffusely infected intestinal tract.

Table 2 shows the distribution of infected digestive areas in these 3 groups.

The 5 sea breams of the SV group were sampled from a pool that had been affected by 2 previous mortal outbreaks due to Enteromyxum leei. The fish are considered here as 'survivors' of these previous outbreaks of myxidiosis. The previously described 'longitudinally cut intestine' protocol was applied to samples from these 5 fish. Four fish had no parasites along their entire intestinal tracts and one was infected along about $50 \%$ of the length of the intestinal mucosa, primarily in the distal and rectal parts of the intestine. fish included in the 'longitudinally cut intestine' protocol.

All fish belonging to the MO group were heavily infected by Enteromyxum leei (Table 1). This group contains 16 "Moribund" fish considered to be at the terminal stage of the disease, with obvious and severe clinical signs associated with severe terminal emaciation. Among the 14 fish included in the 'transversally cut intestine' protocol, only 9 of the 84 examined areas were free of parasites. Eleven of the 14 fish had parasites in all parts of the gut, including the pyloric

\section{Intestinal lesions associated with Enteromyxum leei infection}

\section{Control group}

The normal intestinal mucosa epithelium comprises a single layer of high columnar epithelial cells and contains mucus cells. The nuclei of the epithelial cells are located at the centre or near the basal side of the cells.

The lamina propria of the intestine are made up of loose connective tissue with a well-developed capillary 
Table 2. Distribution of infection in fish included in the 'transversally cut intestine protocol' (for details see 'Materials and methods'); AH: apparently healthy; SS: slightly sick; MO: moribund groups. Total no. examined in parentheses. Chi-squared analysis: comparison between AH and SS or MO groups, ${ }^{*} \mathrm{p} \leq 0.05{ }^{* *} \mathrm{p} \leq 0.01$

\begin{tabular}{|c|c|c|c|c|c|c|c|c|}
\hline \multirow{2}{*}{ Group } & \multirow{2}{*}{$\begin{array}{c}\text { No. of } \\
\text { infected fish }\end{array}$} & \multirow{2}{*}{$\begin{array}{c}\text { No. of } \\
\text { infected fish } \\
\text { with positive } \\
\text { pyloric caeca }\end{array}$} & \multicolumn{5}{|c|}{ No. of infected fish with positive intestinal areas } & \multirow{2}{*}{$\begin{array}{l}\text { Total no. } \\
\text { of infected } \\
\text { areas }\end{array}$} \\
\hline & & & Proximal & $\begin{array}{c}\text { Mid- } \\
\text { proximal }\end{array}$ & $\begin{array}{l}\text { Mid- } \\
\text { distal }\end{array}$ & Distal & Rectum & \\
\hline $\mathrm{AH}$ & $6(10)$ & $0(6)$ & $2(6)$ & $4(6)$ & $4(6)$ & $4(6)$ & $5(6)$ & $19(60)$ \\
\hline SS & $14(14)$ & $11(14)^{*}$ & $14(14)^{* *}$ & $12(14)$ & $13(14)$ & $13(14)$ & $13(14)$ & $76(84)^{* *}$ \\
\hline $\mathrm{MO}$ & $14(14)$ & $12(14)^{* *}$ & $12(14)^{*}$ & $12(14)$ & $14(14)^{*}$ & $12(14)$ & $13(14)$ & $75(84)^{* *}$ \\
\hline
\end{tabular}

network. The muscularis mucosae and submucosa are absent in the intestinal mucosa. The muscularis lies immediately beneath the lamina propria.

In the control group, we consistently observed an irregular and disrupted thin layer of inflammatory cells in contact with the basement membrane in the subepithelial area of the lamina propria, associated with transepithelial figures of exocytosis.

\section{$\mathrm{AH}, \mathrm{SS}$ and $\mathrm{MO}$ groups}

The intestinal areas free of parasites in the infected fish exhibit a normal histological aspect identical to that of the control group.

In parasite-affected areas, the palisade-like arrangement of the affected epithelial layer appears to be disturbed due to numerous pansporoblasts located close to the basement membrane. The affected epithelium has a pseudostratified structure consisting of shortened enterocytes with round nuclei that are irregularly distributed in the cytoplasm (Fig. 3). The epithelial integrity is always preserved, even in the most heavily infected cases, although the affected epithelium layer frequently appears to be separated from the subepithelial connective tissue, possibly as a result of its increased fragility (Fig. 1). The affected intestinal epithelium contains a high number of parasites (Fig. 2).

In the affected intestinal areas, mucus cells appear to be less numerous and smaller, and are even absent in the most heavily affected areas of the mucosa. The number of mucus cells per unit length $(100 \mu \mathrm{m})$ is significantly higher in the non-affected than in the affected areas for a given intestinal location associated with the presence of parasites (median: $4.6 \pm 2.3$ cells $100 \mu^{-1}$ vs. $1.8 \pm 1.1$ cells $\left.100 \mu m^{-1}, \mathrm{n}=24, \mathrm{p}<0.05\right)$, as can be seen in Fig. 5.

In the affected groups, the parasite-affected areas appear to contain a markedly reduced, or even absent, irregular and disrupted thin layer of inflammatory cells in contact with the basement membrane.
The lamina propria shows diffuse inflammatory changes that vary in intensity within and among the groups. We observed a clear severe thickening of the affected villi with partial obliteration of the digestive lumen. Numerous mononuclear and granular eosinophilic inflammatory cells are present, associated with patterns of neovascularisation and diffuse fibrosis (Figs. $6 \& 7$ ). The clear distinction between the epithelium layer and the lamina propria is sometimes obscured by the infiltration of massive inflammatory cells associated with extensive transepithelial exocytosis.

The transition between infected and non-infected areas is always evident as an abrupt boundary in the epithelial and connective tissues, with heavily infected villi adjacent to parasite-free villi showing no inflammatory changes (Fig. 8).

\section{SV group}

In the infected SV group fish, the intestinal areas with parasites show the same histological characteristics as those described in infected fish from the other groups. The non-infected intestinal areas show some specific changes, with a pseudostratified epithelial layer devoid of any pansporoblasts and containing numerous regenerating enterocytes (characterised by a round voluminous nucleus). The goblet cells are large and voluminous, being just as numerous as in the intestinal tracts of the control group. There is an inflammatory cell layer in contact with the basement membrane, which disappears in the parasite-affected areas in infected fish, becoming thicker until it forms a continuous layer (Fig. 9). The lamina propria appears particularly thick and massively infiltrated by inflammatory cells, mainly macrophages. We observed numerous fibroblasts in a dense connective tissue composed of collagen and a large network of new capillary blood vessels, mainly at the end of villi. The neovascularisation and fibrosis pattern indicate a healing process. 

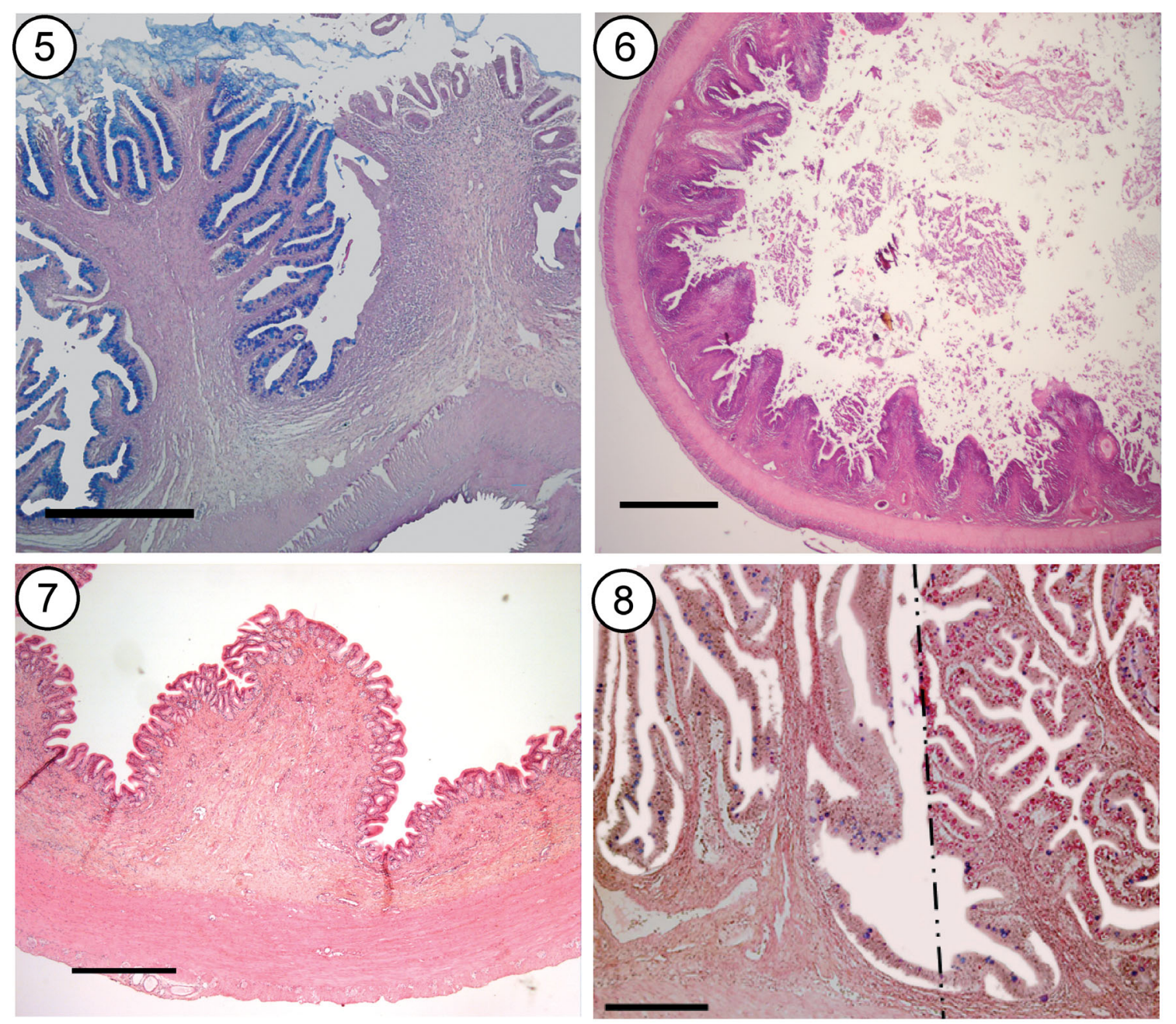

Figs. 5 to 8. Enteromyxum leei infecting Sparus aurata. Fig. 5. Intestine of a sea bream infected by E. leei. Infected villi show a significant decrease in the number of mucus cells associated with the parasites, as clearly shown by PAS-Alcian blue staining. The non-infected villus (left) is strongly stained blue, corresponding to goblet cells, whereas the infected villus (right) has no blue staining. Scale bar $=400 \mu \mathrm{m}$. Fig. 6. Intestine of a sea bream heavily infected by E. leei. Lesions of myxidiosis are typical of severe enteritis with atrophy and blunting of villi as well as fibrotic lamina propria. HES. Scale bar $=1000 \mu$ m. Fig. 7. Detail of Fig. 6 showing atrophy and blunting of villi with fibrosis of the lamina propria. Inflammation is limited to the foci of mononucleated inflammatory cells. HES. Scale bar $=500 \mu \mathrm{m}$. Fig. 8. Transition between infected intestinal villus and non-infected intestine villus. The infected villi contain a very large number of parasites (stained magenta on the villi to the right of the dot-dash line), showing an abrupt transition between the 2 areas with no progressive transition in the intensity of the Parasites. PAS-Alcian blue. Scale bar $=350 \mu \mathrm{m}$

\section{Histological changes of the extra-digestive organs}

\section{Liver}

The liver shows moderate to severe glycogenic and fatty accumulation, with glycogenic accumulation in some vacuolated nuclei in fish of all groups, including the control. The intra-hepatic biliary ducts are moderately infected by $E$. leei in 1 fish of the SS group and in 1 fish of the MO group. The proximal part of the gall bladder, which contains villi similar to intestinal villi, is infected by parasites in 2 fish from the SS and MO groups, but the distal part of the gall bladder, being devoid of villi, is always free of parasites. We failed to observe any inflammatory changes in the gall bladder associated with the presence of parasites.

\section{Exocrine pancreas}

The exocrine pancreas sheets included in the hepatic tissue show a severe topographic regression in 7 fish from the SS group and 9 fish from the MO group. The pancreas regression is associated with an evident atro- 

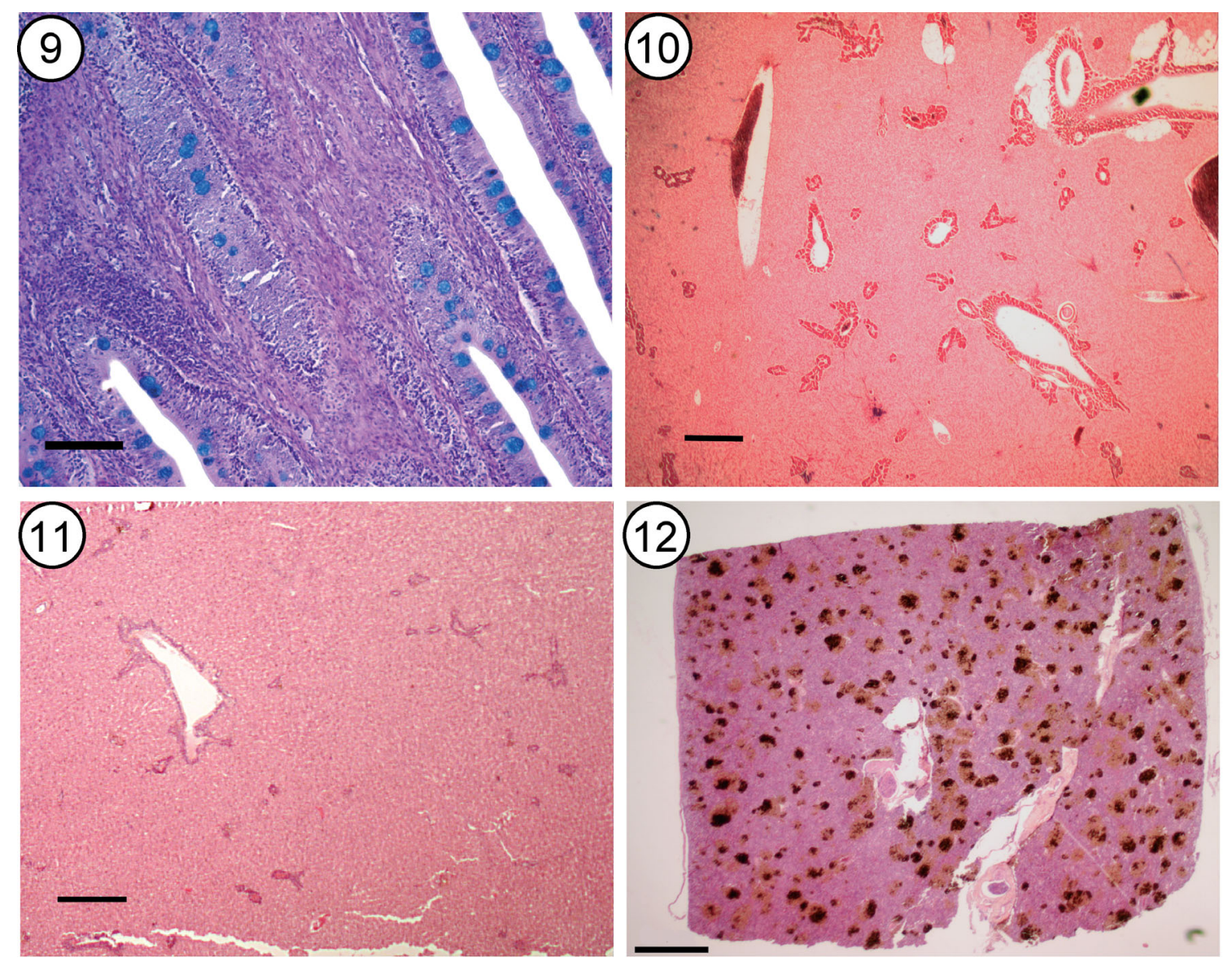

Figs. 9 to 12. Fig. 9. Enteromyxum leei infecting Sparus aurata. Villi from the intestine of a 'Survivor' SV group fish showing abundant granulation tissue characterised by diffuse fibrosis of the lamina propria and an infiltration of mononucleated inflammatory cells. The subepithelial inflammatory layer appears larger and more continuous in survivor fish than in control fish. No parasites were found in the intestine of most fishes from the SV group. PAS-Alcian blue. Scale bar $=50 \mu \mathrm{m}$. Fig. 10. S. aurata. Hepatopancreas of control fish showing normal pancreas extension around biliary tracts and around vessels. HES. Scale bar $=500 \mu \mathrm{m}$. Fig. 11. E. leei infecting $S$. aurata. Hepatopancreas of fish severely infected by E. leei showing marked atrophy of the pancreatic tissue. Scale bar $=500 \mu \mathrm{m}$. HES. Fig. 12. E. leei infecting $S$. aurata. Spleen of an infected fish from the 'Slightly sick' group. Melanomacrophagic centres (MMC) are greatly increased in size and number with deposition of ferric pigments (brownish coloration) around the brown-black MMC. HES. Scale bar $=500 \mu \mathrm{m}$

phy of the exocrine cells that are almost completely devoid of zymogen granules. In the most severe cases, the exocrine pancreatic tissue completely disappears (cf. Figs. 10 \& 11; control vs. infected tissues, respectively). In the SV group, the location and extent of the pancreatic tissue are identical to the control group.

\section{Spleen}

In comparison with the spleens of the control group, the number and size of melanomacrophagic centres (MMC) loaded with iron-bearing pigments (positively stained by Perls' technique) progressively increases from the AH, through the SS to the MO group. (Fig. 12)
The MMC account for most of the splenic tissue in the fish of the MO group. In the SV group, the number and size of MMC are identical to those observed in the $\mathrm{AH}$ group.

\section{DISCUSSION}

The myxosporean parasite observed in the present study was identified as Enteromyxum leei, and has previously been described in Mediterranean farmed sea bream (Diamant 1992, Diamant et al. 1994) and other cultured Mediterranean fish species (Le Breton \& Marques 1995, Padros et al. 2001). Studies have shown that water temperature determines the multiplication 
of parasites in infected fish, leading to the clinical expression of the disease in the most heavily infected animals. The minimum temperature for developing the disease in infected fish farms varies from $18^{\circ} \mathrm{C}$ (Le Breton \& Marques 1995) to $22^{\circ} \mathrm{C}$ (Rigos et al. 1999). In the infected farm studied here, the water temperature is artificially maintained between 15 and $25^{\circ} \mathrm{C}$ throughout the year (i.e. $10^{\circ} \mathrm{C}$ higher than the temperature of the natural coastal sea water) by using reprocessed water from a nuclear power station. The high water temperature not only improves the weight gain of the fish, but also increases the possibility for this parasite to develop in infected pools (Le Breton \& Marques 1995, Rigos et al. 1999). Since 2001, outbreaks of myxidiosis in French fish farms have been exclusively observed during the summer months, when the water temperature rises above $20^{\circ} \mathrm{C}$. Neither the intermediate host nor the source of infection has yet been identified in the fish farm discussed here.

Enteromyxum leei shows a clear tropism for the intestine. The parasites are primarily observed in the intestinal mucosa from the pyloric caeca to the rectum. Unlike Le Breton \& Marques (1995), we never found any parasites in the stomach. Extra-digestive locations are unusual, as only 4 of the 52 fish developed E. leei on the mucosa of hepatic biliary ducts and gall bladder. Such extra-digestive locations (biliary duct epithelium) have already been described by Le Breton \& Marques (1995) and can be explained by the common embryological origin of the intestine and biliary ducts and by the close proximity between these 2 structures.

The histological examination of sea breams without any clinical sign of the disease (AH group) sampled from affected pools showed that a considerable number of apparently healthy fish (around $40 \%$ ) were free of parasite infection despite being in contact with potentially infected fish in the same pool. In the apparently healthy infected fish, the different parts of the intestinal tract were never simultaneously infected. The intestinal infection seemed to proceed by a single or few primary foci located in the intestine. These foci are likely to extend from cell to cell by close contact between infected cells and non-infected cells, as indicated by the severely infected intestinal area bordering non-infected areas. The rectum and posterior parts of the intestine remained free of parasites in some affected fish (5 out of 10 in the AH group). This suggests that the technique of scraping the rectal mucosa commonly used for diagnoses of myxidiosis in asymptomatic fish can potentially produce numerous false negative results (50\% in the AH group).

The clinical signs of myxidiosis appear during the terminal stage of the disease, and only if associated with a heavy and diffuse - almost generalised - infection of the intestinal tract. The very small differences (not statistically significant) in the extension and severity of the parasite infection and associated lesions between the SS and MO groups may indicate the rapid spread of parasite infection within an animal.

The focal parasite infection of the intestinal mucosa in asymptomatic fish and the heavy diffuse and almost generalised infection of the intestinal tract in all of the symptomatic fish are consistent with a direct route of transmission along the entire intestinal mucosa from a few limited primary infected areas. The 2 'cell in a cell' stages of unknown origin observed in the mature pansporoblasts are possibly responsible for the direct intestinal extension of the parasites and may promote a rapid severe infection of the same host by spread of the developmental stages (Diamant 1997, Yasuda et al. 2002). There could also be some way for direct fishto-fish transmission, which may be considered as a possible alternative transmission route to the myxosporean/ actinosporean alternating life cycles (Benajiba \& Marques 1993).

The parasitic stages observed in histological sections correspond to the sporogonic stages which seem to be located between epithelial cells as described by Diamant et al. (1994) studying the same sporogonic stages (sporoblasts and spores) by transmission electronic microscopy. They described a disporic stage wedged between the basal parts of the epithelial cells of the intestinal mucosa. The spore shape and dimensions are identical to those previously described in sea bream (Diamant 1992, Diamant et al. 1994, Sakiti et al. 1996, Le Breton \& Marques 1995). Although it is difficult to identify the parasite by HES staining, other stains, such as PAS-Alcian blue and Gram techniques, allow the parasite structure to be more clearly determined and thus improve identification. PAS-Alcian blue staining clearly distinguishes the pansporoblasts (stained magenta) from the apical cytoplasm of the goblet cells (stained blue). Thus, it is the simplest method for identifying the myxosporean and estimating the degree of digestive parasite infection.

A marked decrease in the number of goblet cells in affected areas is demonstrated in our study by comparative counting of mucus cells between affected and non-affected areas of the same fish. We observed a severe disruption of the epithelium structure, with local detachment frequently occurring in heavily infected fish. In contrast to Tun et al. (2002), who considered the epithelial detachment as pathologic, due to the accumulation of necrotic cells under the epithelium layer, we consider the local detachment of the epithelium to be an indirect result of the intestinal fragility associated with a massive parasite infection.

The main lesions associated with the intestinal parasite infection consist of a subacute to chronic severe diffuse inflammatory infiltration of the lamina propria 
by mononuclear and granular eosinophilic cells, associated with fibrosis and neovascularisation. This is an evident intestinal host reaction, associated with the increasing size and number of spleen melanomacrophagic centres, although this seems to be more limited than the host reaction described by Tun et al. (2002) in tiger puffer fish infected by Leptotheca fugu, Enteromyxum fugu (previously Myxidium fugu) and Myxidium sp.

The cause of this severe atrophy of pancreatic tissue is not clear and may be the result of prolonged fasting in heavily infected fish. The clinical signs may be partly explained by the severe intestinal changes associated with the very large decrease in goblet cells, along with the almost total pancreatic atrophy. In particular, these changes may account for the emaciation and increase in feed conversion ratio, following on from the poor absorption of nutrients and failure of osmoregulation.

In almost all examined cases, fish considered to be survivors from a pool previously infected by Enteromyxum leei have digestive tracts showing a natural elimination of the parasites, with a host reaction and some evidence of healing. These observations suggest that some infected fish may acquire immunity, with the possible spontaneous elimination of the parasites and potential recovery of the intestinal mucosa. This appears consistent with the spontaneous evolution of the disease in the studied fish farm.

Acknowledgements. The authors thank S. Leuillet for his assistance with the statistical analysis, and M. S. N. Carpenter for improving the English.

\section{LITERATURE CITED}

Benajiba MH, Marques A (1993) The alternation of actinomyxidian and myxosporidian sporal forms in the development of Myxidium giardi (parasite of Anguilla anguilla) through oligochaetes. Bull Eur Assoc Fish Pathol 13: 100-103

Close B, Banister K, Baumans V, Bernoth EM and others (1997) Recommendations for euthanasia of experimental animals: Part 2. Laboratory Anim 31:1-32

Diamant A (1992) A new pathogenic histozoic Myxidium (Myxosporea) in cultured gilthead sea bream Sparus aurata L. Bull Eur Assoc Fish Pathol 12:68-69

Diamant A (1997) Fish-to-fish transmission of a marine myxosporean. Dis Aquat Org 30:99-105

Editorial responsibility: Dieter Steinhagen,

Hannover, Germany
Diamant A, Lom J, Dykova I (1994) Myxidium leei n. sp., a pathogenic myxosporean of cultured sea bream Sparus aurata. Dis Aquat Org 20:137-141

El Matbouli M, Hoffmann R (1993) Myxobolus carassii Klokaceva, 1914 also requires an aquatic oligochaete Tubifex tubifex as an intermediate host in its life cycle. Bull Eur Assoc Fish Pathol 13:189-192

Grassé P, Lavette A (1978) La Myxosporidie Sphaeromyxa sabrazesi et le nouvel embranchement des myxozoaires (Myxozoa). Recherches sur l'état pluricellulaire primitif et considérations phylogénétiques. Ann Sci Nat Zool Paris 20:193-285

Le Breton A, Marques A (1995) Occurrence of an histozoic Myxidium infection in 2 marine cultured species: Puntazzo puntazzo C. and Pagrus major. Bull Eur Assoc Fish Pathol 15:210-212

Lom J, Dykova I (1995) Myxosporea (phylum Myxozoa) In: Woo PTK (ed) Fish diseases and disorders, Vol 1: Protozoan and metazoan infections. CAB International, Wallingford, p 97-139

Padrós F, Palenzuela O, Hispano C, Tosas O, Zarza C, Crespo S, Alvarez-Pellitero P (2001) Myxidium leei (Myxozoa) infection in aquarium-reared Mediterranean fish species. Dis Aquat Org 47:57-62

Palenzuela O, Redondo MJ, Alvarez-Pellitero P (2002) Description of Enteromyxum scophthalmi gen. nov. sp. (Myxozoa), an intestinal parasite of turbot (Scophthalmus maximus L.) using morphological and ribosomal RNA sequence data. Parasitology 124:369-379

Rigos G, Christophilogiannis P, Yiagnisi M, Andriopoulou A, Koutsodimou M, Nengas I, Alexis M (1999) Myxosporean infections in Greek mariculture. Aquac Int 7:361-364

Sakiti N, Tarer V, Jacquemin D, Marquès A (1996) Présence en Méditerranée occidentale d'une Myxosporidie histozoïque pathogène dans les élevages de Daurade, Sparus aurata L. Ann Sci Nat Zool Paris 17:123-127

Tarer V, Sakiti N, Le Breton A, Marques A (1996) Myxidium leei, myxosporidie pathogène chez les sparidés en aquaculture en méditerranée. Ichtyophysiol Acta 19: $127-139$

Tun T, Ogawa K, Wakabayashi H (2002) Pathological changes induced by three myxosporeans in the intestine of cultured tiger puffer, Takifugu rubripes (Temminck and Schlegel). J Fish Dis 25:63-72

Wolf K, Markiw ME (1984) Biology contravenes taxonomy in the Myxozoa: new discoveries show alternation of invertebrate and vertebrate hosts. Science 225:1449-1452

Yanagida T, Nomura Y, Kimura T, Fukuda Y, Yokoyama H, Ogawa K (2004) Molecular and morphological redescriptions of enteric myxozoans, Enteromyxum leei (formerly Myxidium sp. TP) and Enteromyxum fugu comb. n. (syn. Myxidium fugu) from cultured tiger puffer. Fish Pathol 39:137-143

Yasuda H, Ooyama T, Iwata K, Tun T, Yokoyama H, Ogawa K (2002) Fish to fish transmission of Myxidium spp. (Myxozoa) in cultured tiger puffer suffering from emaciation disease. Fish Pathol 37:29-33

Submitted: March 19, 2007; Accepted: May 24, 2007

Proofs received from author(s): April 11, 2008 